\title{
Prenatal cigarette smoke exposure slightly alters neurobehavioral development in neonatal rats: Implications for developmental origins of health and disease (DoHAD)
}

B. MAMMEL ${ }^{1,2 *}$ (D) T. KVÁRIK ${ }^{1,2}$, ZS. SZABÓ ${ }^{2}$, J. GYARMATI $^{1}$, T. ERTL $^{1}$, J. FARKAS ${ }^{2}$, ZS. HELYES ${ }^{3,5}$, T. ATLASZ ${ }^{2,4}$, D. REGLÖDI ${ }^{2 \dagger}$ and P. KISS ${ }^{2 \dagger}$

${ }^{1}$ Department of Obstetrics and Gynecology, Department of Neonatology, University of Pécs, Pécs, Hungary

${ }^{2}$ Department of Anatomy, University of Pécs Clinical Centre, Pécs, Hungary

${ }^{3}$ Department of Pharmacology and Pharmacotherapy, University of Pécs Medical School, Pécs, Hungary

${ }^{4}$ Department of Sportbiology, University of Pécs, Pécs, Hungary

${ }^{5}$ Szentágothai Research Center, University of Pécs, Pécs, Hungary

Received: November 22, 2019 • Accepted: December 04, 2019

Published online: April 23, 2020

(C) 2020 Author(s)

\begin{abstract}
Numerous studies indicate that smoking during pregnancy exerts harmful effects on fetal brain development. The aim of this study was to determine the influence of maternal smoking during pregnancy on the early physical and neurobehavioral development of newborn rats. Wistar rats were subjected to whole-body smoke exposure for $2 \times 40 \mathrm{~min}$ daily from the day of mating until day of delivery. For this treatment, a manual closed-chamber smoking system and 4 research cigarettes per occasion were used. After delivery the offspring were tested daily for somatic growth, maturation of facial characteristics and neurobehavioral
\end{abstract}

* Corresponding author. Department of Obstetrics and Gynecology, Department of Neonatology, University of Pécs, Szigeti Street 12, 7621, Pécs, Hungary. Tel.: +36 72/536-000/31627. E-mail: mammel.barbara@gmail.com

$\dagger$ These authors contributed equally. 
development until three weeks of age. Motor coordination tests were performed at 3 and 4 weeks of age. We found that prenatal cigarette smoke exposure did not alter weight gain or motor coordination. Critical physical reflexes indicative of neurobehavioral development (eyelid reflex, ear unfolding) appeared significantly later in pups prenatally exposed to smoke as compared to the control group. Prenatal smoke exposure also resulted in a delayed appearance of reflexes indicating neural maturity, including hind limb grasping and forelimb placing reflexes. In conclusion, clinically relevant prenatal exposure to cigarette smoke results in slightly altered neurobehavioral development in rat pups. These findings suggest that chronic exposure of pregnant mothers to cigarette smoke (including passive smoking) results in persisting alterations in the developing brain, which may have long-lasting consequences supporting the concept of developmental origins of health and disease (DoHAD).

\section{KEYWORDS}

prenatal, smoking during pregnancy, tobacco, neurobehavioral development, rat

\section{INTRODUCTION}

Numerous studies indicate that smoking during pregnancy may have harmful effects on the newborn. Perinatal exporure to smoking can affect fetal development, resulting in delayed cognitive and motor development, retarded locomotor behavior, and an increase in the incidence of psychiatric abnormalities [12, 13, 30, 31].

Tobacco smoke contains thousands of chemicals [21], and many of these are potentially toxic. The major components in tobacco smoke that have been shown to interfere with brain development are carbon monoxide and nicotine [9]. Nicotine crosses the placenta, enters the fetal circulation and accumulates in the fetal compartments from as early as 7 weeks of gestation in both active and passive smokers $[19,25,33]$. In rats, nicotine has negative effects on brain and lung development $[6,17,35,42]$. Prenatal nicotine exposure has also been shown to affect the contractility of the uterine arteries, leading to decreased uterine blood flow in animals. Damaging effects have also been shown in first-trimester human fetal brain cell cultures [18]. Nicotine binds to the cholinergic receptors during brain development causing cell death or structural alteration in certain brain areas, which leads to neurobehavioral and functional impairment in offspring [45]. Carbon monoxide also crosses the placenta, where it binds to hemoglobin, producing carbohaemoglobin, which limits oxygen delivery to the tissues [19]. Therefore, exposure to maternal smoking may lead to fetal hypoxia and ischemia, which affect brain development. Perinatal hypoxia-ischemia increases the risk of future behavioral and neurological deficits. Locomotor ability, learning and memory deficits have been described in juvenile and adult rats subjected to a hypoxic-ischemic insult in the perinatal period $[19,23,24]$. Metals, including lead, cadmium and mercury, which are all neurotoxins, have also been detected in cigarette smoke [4]. Cigarette smoke has well-known damaging effects at all ages, leading to cancerogenesis, vasculopathies and accelerated aging [27, 46]. Recent studies reported that parental smoking during maternal pregnancy may be associated with an increased risk of childhood overweight as well as obesity in adulthood leading to hypertension and other cardiovascular diseases $[2,8,11,15,51]$. Maternal smoking during pregnancy has also been 
associated with increased risk of wheezing, asthma, airway hyper responsiveness, impaired lung function, and bronchitis [7].

In human epidemiological studies it is difficult to prove that smoking during pregnancy has the potential to cause fetal brain damage, because of the many co-variables that operate together with smoking. Such factors are low socio-economic status, poor prenatal care or co-abuse of different other drugs. Using an animal model, these problems can be resolved. A number of methods for the administration of tobacco smoke during pregnancy have been employed to assess the potential of nicotine and other cigarette smoke components in causing altered neuronal development in rodents. A recent study demonstrates significant increase in locomotor activity that was observed as early as 4 weeks of age persisting through 4 months of age in a mouse model [50]. These findings are similar to reports in humans where investigators found an increased risk for attention-deficit hyperactivity disorder, aggression and other childhood behavior problems $[5,8]$. However, we know little about the effects of prenatal smoke exposure on the early development of the offspring.

Development is reflected among others by the maturation of neurological reflexes and motor coordination. The appearance of certain neurological reflexes is known to be influenced by various factors $[20,43,44]$. Although the maturation of neurological reflexes represents hallmarks of nervous system development $[1,2,10,15,20,43,44]$, relatively little is known about the maturation of these reflexes following prenatal exposure to smoking. The aim of the present study was to investigate the influence of maternal smoking during pregnancy on the early physical and neurobehavioral development of newborn rats. Our hypothesis was that maternal smoking during pregnancy interferes with development and therefore results in a delayed physical and motor development and changed locomotor behavior, which could be traceable with a battery of tests at early stages of the life of rat pups.

\section{MATERIALS AND METHODS}

\section{Animals}

Male and female Wistar rats aged 2-4 month were chosen to be mated, and randomly distributed in smoking and non-smoking group. All animals were maintained under $12 \mathrm{~h}$ light/ dark cycle with free access to food and water. Animal housing, care and application of experimental procedures were in accordance with the institutional guidelines under approved protocols (No.: BA02/2000-17/2012, University of Pécs). Two female rats and a male rat were mated in one cage. Animals were exposed to whole-body smoke exposure for $2 \times 40$ min daily from mating until delivery. A TE2 closed-chamber manual smoking system (Teague Enterprises, Woodland) and 4 3R4F research cigarettes (College of Agriculture, University of Kentucky, Lexington, KI) were used to deliver cigarette smoke to animals. This model represents an animal model for passive smoking. Offspring of these female rats were the prenatal smoke-exposed group (PSE).

The control group (2 females and 1 male rat) was housed in another chamber of the same type, but were not exposed to cigarette smoke. Altogether four litters were used, 2 PSE and 2 control. Animals were cross-fostered immediately after birth to minimize litter differences and effects of possible changes in maternal behavior due to smoke exposure. 


\section{Neurobehavioral testing}

The assessment of neurobehavioral development was started on the first postnatal day and was carried out daily between 6 and 9 am by the same person (BM) until postnatal day 21 (PND), in a blinded fashion. Neurobehavioral maturation and development of motor coordination were tested using standardized procedures. Weight was measured daily until 3 weeks of age, as well as maturation of physical characteristics such as eye opening, incisor eruption and ear unfolding. Pups were tested for the following signs and reflexes: (1) Surface righting reflex: rats were placed in supine position and the time in seconds to turn over to prone position and place all four paws in contact with the surface was recorded. (2) Negative geotaxis: animals were placed head down on an inclined grid $\left(45^{\circ}\right)$ of $30 \mathrm{~cm}$. The forelimbs of the pups were placed in the middle of the grid. The day they began to turn around and climb up the board with their forelimbs reaching the upper rim was observed. In cases the animal did not turn around and climb up the board within the observed $30 \mathrm{~s}$, the test was considered negative. From the day of the appearance of the negative geotaxis, the time in seconds to reach the upper end of the board was recorded daily. (3) Crossed extensor reflex: the left rear paw was pinched and the animal was observed for the extension of the right leg. The day of disappearance of the crossed extensor reflex in its pure form, when it was replaced by a more complex behavioral response, was noted. (4) Sensory reflexes: the ear and the eyelid were gently touched with a cotton swab and the first day of the ear twitch reflex and the contraction of the eyelid were recorded. (5) Limb placing: the back of the forepaw and the hind paw was touched with the edge of the bench while the animal suspended, and the first day of lifting and placing the paws on the table was noted. (6) Limb grasp: the foreand hindlimbs were touched with a thin rod, and the first day of grasping onto the rod was recorded. (7) Gait: the animals were placed in the center of a white paper circle of $13 \mathrm{~cm}$ in diameter, and the day they began to move off the circle with both forelimbs was recorded. In cases when the animal did not leave the circle for $30 \mathrm{~s}$, the test was considered to be negative. From the day of the appearance, the time in seconds to move off the circle was recorded daily. (8) Auditory startle: the first day of the startle response to a clapping sound was observed. (9) Air righting: the pups were dropped head down onto a bed of shavings from a height of $50 \mathrm{~cm}$, and the day of first landing on four feet was recorded.

\section{Motor coordination test}

Rat pups were tested for the development of more complex motor behavior once a week on the third and fourth week of age.

Grid walking: rats were placed on a stainless steel grid floor $(20 \mathrm{~cm} \times 40 \mathrm{~cm}$ with mesh size of $4 \mathrm{~cm}^{2}$ ) elevated $1 \mathrm{~m}$ above the floor. For a 1-min observation period, the total number of steps was counted. Foot-fault test: The number of foot-fault errors, when the animals misplaced a forelimb or hindlimb that it fell through the grid, was also recorded during a 1-min period.

\section{Open-field activity}

Animals were observed for locomotor and spontaneous exploratory behavior in an open field at 5 weeks of age as previously described [24, 28, 34]. Pups were placed in an open field consisting of a $42 \mathrm{~cm} \times 42 \mathrm{~cm}$ box with $21 \mathrm{~cm}$ high walls around. Rats were placed individually in the center, always facing the same direction, and were video-recorded for $5 \mathrm{~min}$. Recordings were 
evaluated in blinded fashion. The following parameters of locomotor activity were measured: head lifting, grooming, walking by the wall, or time spent the middle of the box. The time spent in the first zone next to the wall was also measured.

\section{Statistical analysis}

Data are expressed as mean \pm standard error of the mean (SEM). The results in appearance of physical and neurological signs as well as daily weights were compared with Student's $t$-test. Improvements in daily performance in righting reflex, negative geotaxis and gait were evaluated by two-way ANOVA repeated measures, whereas daily values were compared by $t$-test. Statistical significance is reported at $P \leq 0.05$.

\section{RESULTS}

Only one pup from the prenatal smoke-exposed (PSE) group died on the first day. No animal died in the control group. There was no significant difference in weight gain between the prenatally smoke-exposed and the control group (Fig. 1).

\section{Neurological signs and reflexes}

Most neurological signs appeared slightly later in prenatally smoke-exposed pups. The delay of ear unfolding was significant. In addition, several neurological reflexes, such as hind limb placing, eyelid reflex and forelimb grasp developed noticeably later (Fig. 2). PSE animals had significantly better performances in negative geotaxis throughout the test period (Fig. 3).

\section{Motor coordination}

Among the many motor coordination tests available, the most reliable indicator in our previous studies had been the grid walking/foot fault test [14, 28, 32]. In the grid-walking test, PSE animals had significantly lower total number of steps on the third week of age (Fig. 4). The number of foot faults in ratio to the total number of steps was lower in the PSE group in case of

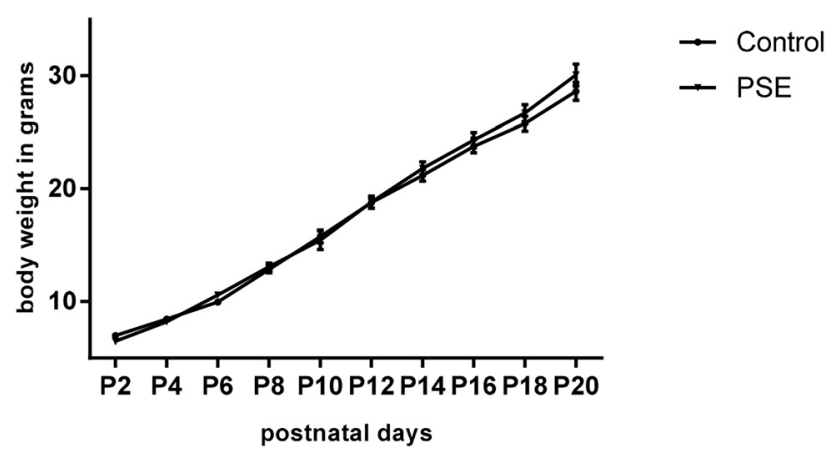

Fig. 1. Weight gain of control and prenatal smoke pups; Control vs. PSE (prenatally smoke-exposed). Values are expressed as average grams \pm SEM 


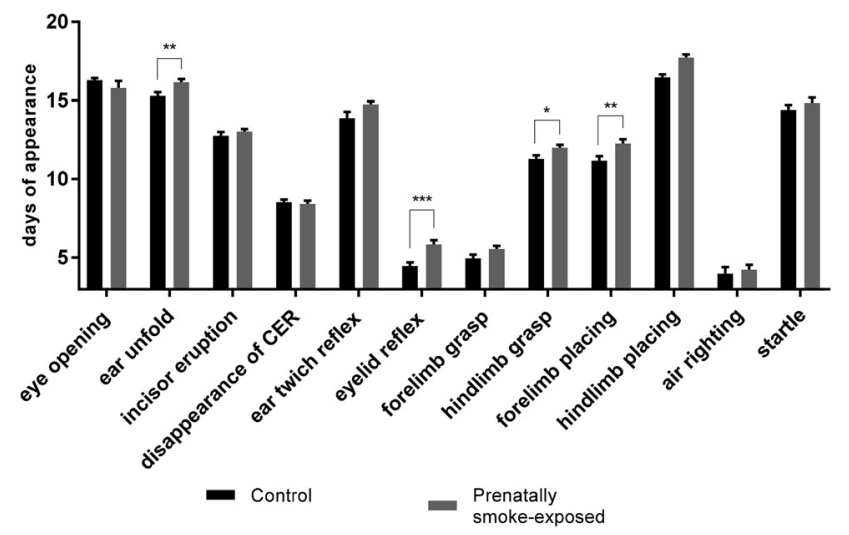

Fig. 2. Appearance of somatic signs and neurological reflexes. Values are expressed as average days \pm SEM. ${ }^{*} P<0.05 ; * * P<0.01 ; * * * P<0.001$

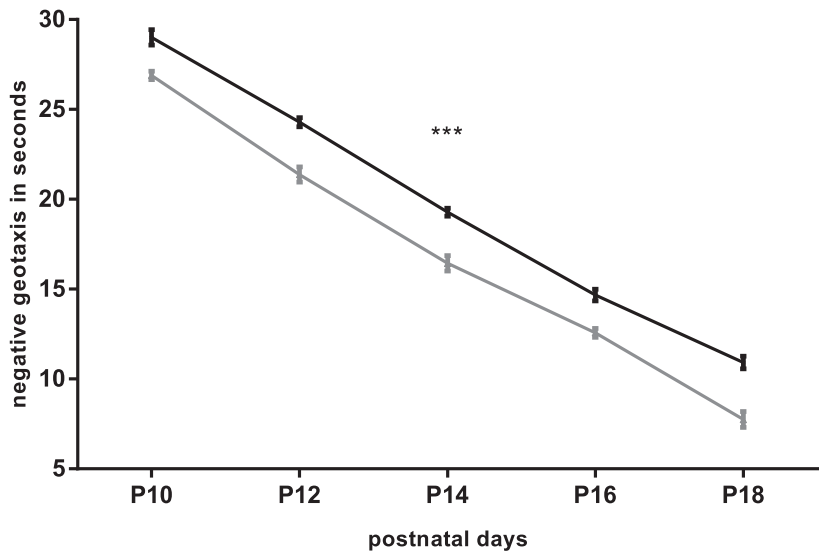

- Control $\quad-$ Prenatally smoke-exposed

Fig. 3. Daily performance of negative geotaxis reflex of control and PSE pups. Values are expressed as average seconds \pm SEM. $* * * P<0.0005$

the left and right forelimb and the right hind limb on the third week of age. PSE pups made significantly less foot faults in ratio to the total number of steps with both of their hind limbs at 4 weeks of age.

\section{Open field activity}

In the open field test, general activity and movement pattern did not show gross differences between the groups. The number of head lifting decreased in both groups during the trials 

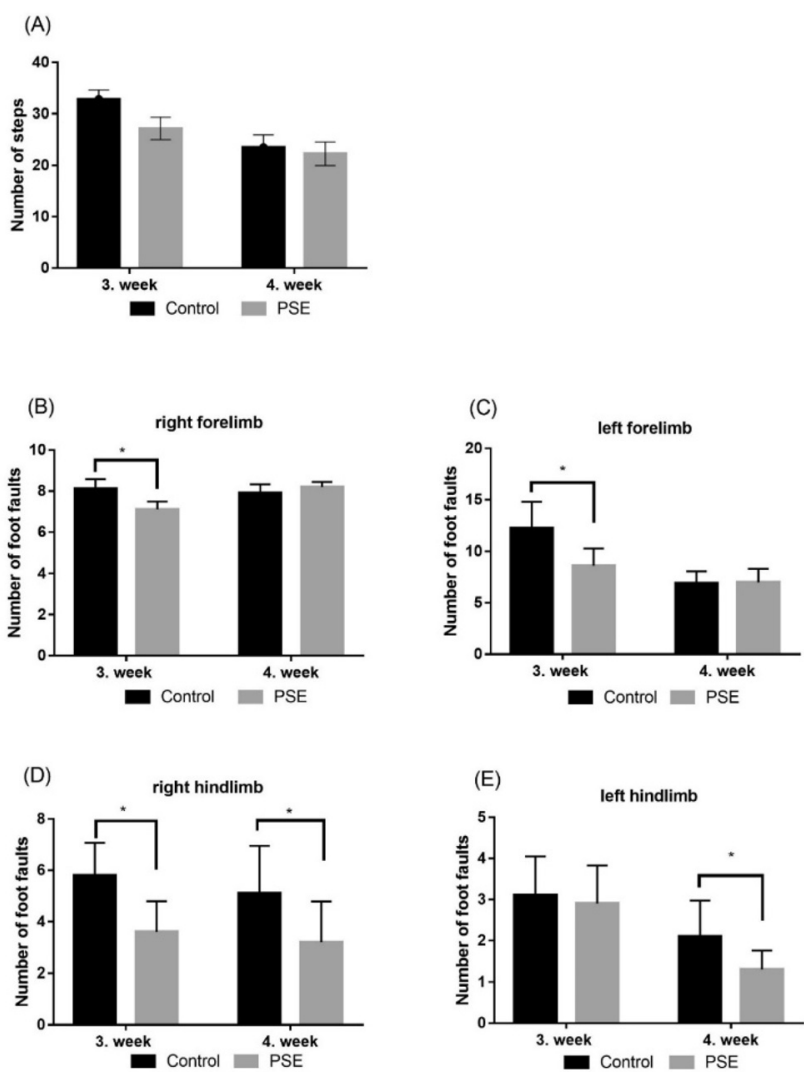

Fig. 4. Performances in motor coordination tests of control and prenatally smoke-exposed (PSE) animals. (A) Number of total steps in the grid-walking test. (B) Number of foot faults in ratio to number of total steps with the right forelimb. (C) Number of foot faults in ratio to number of total steps with the left forelimb. (D) Number of foot faults in ratio to number of total steps with the right hind limb. (E) Number of foot faults in ratio to number of total steps with the left hind limb. Values are expressed as average \pm SEM. $* P<0.05$

monitoring but no significant differences could be observed between the control and PSE animals. The time spent with grooming increased with every trial, but there was no difference between the two groups. Similar pattern was observed in the speed and movement pattern of the animals: no significant differences were found in total rest time, walking near the wall or a subject in the center of the box (data not shown).

\section{DISCUSSION}

Perinatal injuries and exposure to toxic agents can lead to many long-term damages, even at older age, but little is known about immediate consequences [26, 37, 38, 40, 47]. 
The prenatal period is one of the critical age windows - in addition to early postnatal life and adolescence - that is open to plastic changes and the influence of external agents [34]. Adverse effects such as chemicals in tobacco smoke can cause severe damage with permanent disabilities. Most studies regarding the negative effects of perinatal treatments focus mainly on long-term effects, and most examinations are carried out on adult animals following perinatal injuries. Less data is available on short-term effects and early neurobehavioral changes.

In the present study we showed detectable changes in the maturation of physical parameters and neurologic development of rat pups after prenatal smoking. Although we did not find any difference in birth weight, growth was slightly more dynamic in the prenatally smok-exposed group. Certain physical reflexes and signs of PSE pups, like eyelid reflex and ear unfolding appeared later compared to the control pups. We also observed a delay in reflexes, indicating neural maturity, like in case of limb placing and grasping reflexes. Maternal exposure to tobacco smoke may lead to some neuromuscular and behavioral deficits in nursing pups.

On the other hand, we observed a remarkably better motility in PSE pups in negative geotaxis testing. In addition, the number of foot faults was lower in case of the pups prenatally exposed to smoke. These findings are in agreement with several studies demonstrating detrimental effects of prenatal smoke exposure $[12,31]$. Other studies indicate that rats exposed to variable prenatal stress evolved, in addition to several behavioral anomalies, increased locomotor behavior and stereotypic-like behaviors [49]. In contrast to these, several studies where only one important component of tobacco smoke, nicotine was exposed to pregnant rats, rat pups showed deficits in righting reflexes and the negative geotaxis test $[36,41]$. During prenatal smoke exposure only a small amount of nicotine crosses the placenta, which means a lower risk to neurobehavioral deficits. These findings suggest that PSE has more similarities with prenatal stress exposure.

Several toxic agents have been shown to cause altered neurobehavioral development [39]. One of the mechanisms of neurotoxicity is excitotoxicity caused by excessive levels of monosodium glutamate (MSG). MSG is a food additive widely used as a flavoring substance. In contrast to adults, the immature blood-brain barrier in new born rat pups allows significant transport of MSG into the nervous system. In our previous experiments, where MSG was given on postnatal days 1,3,5,7, and 9, mortality was markedly higher in MSG-treated pups than in the control group [28]. We found minor delays in the appearance of certain neurological reflexes (forelimb placing and grasping, air righting) and body weight and length were also significantly lower in the MSG-treated pups. MSG treatment also caused worse performance in motor coordination tests.

Both pre- and postnatal stress models are widely used in neuro-endocrinological and psychiatric research $[3,16,29]$. These stress models may provide important correlation with human psychiatric disorders. Maternal separation of rat pups is a well-known model for early life events as it induces long-lasting changes in several stress-related systems [16, 34]. Interestingly, weight gain and reflex development was faster in male pups exposed to perinatal stress. We found a subtle enhancement in male rats, but only slight delay in females.

Previously we have also shown major retardation in the development of neurological signs and reflexes in animal models of perinatal pathologies like asphyxia and hypoxia. Almost all reflexes were delayed and showed a severe, from one to even 4-day delay, and the appearance of some physical signs was also delayed $[22,28]$. Compared to these other insults, prenatal exposure to smoking did not result in such marked differences. We suppose that maternal smoking during pregnancy may cause an increased vulnerability to harmful impacts later in adulthood. 
According to the "Developmental Origins of Health and Disease (DOHaD)" hypothesis, which posits that environmental exposures during multiple sensitive periods of development (especially the in utero period) have a lasting impact on health and disease risk, we suppose that maternal smoking during pregnancy may cause an increased vulnerability to harmful impacts later in adulthood [48].

Conflict of interest: No conflict of interest.

\section{ACKNOWLEDGMENTS}

Funding: Grant numbers GINOP-2.3.2-15-2016-00050 “PEPSYS," MTA-TKI14016; EFOP3.6.3-VEKOP-16-2017-00009, EFOP-3.6.1.-16-2016-00004 Comprehensive Development for Implementing Smart Specialization Strategies at the University of Pécs; TAMOP 4.2.4.A/2-11-12012-0001, EFOP-3.6.3-VEKOP-16-15 2017-00008, OTKA: NKFIH 129190; NAP2017-1.2.1NKP-2017-00002., OTKA PD109644, FIKP II.

\section{REFERENCES}

1. Al Mamun A, Lawlor DA, Alati R, O'Callaghan MJ, Williams GM, Najman JM. Does maternal smoking during pregnancy have a direct effect on future offspring obesity? Evidence from a prospective birth cohort study. Am J Epidemiol 2006; 164: 317-25, https://doi.org/10.1093/aje/kwj209.

2. Altman J, Sudarshan K. Postnatal development of locomotion in the laboratory rat. Anim Behav 1975; 23: 896-920, https://doi.org/10.1016/0003-3472(75)90114-1.

3. Baier CJ, Katunar MR, Adrover E, Pallares ME, Antonelli MC. Gestational restraint stress and the developing dopaminergic system: an overview. Neurotox Res 2012; 22(1): 16-32, https://doi.org/10.1007/s12640-0119305-4.

4. Bernhard D, Rossmann A, Wick G. Metals in cigarette smoke. IUBMB Life 2005; 57(12): 805-9, https://doi. org/10.1080/15216540500459667.

5. Bruin JE, Gerstein HC, Holloway AC. Long-term consequences of fetal and neonatal nicotine exposure: a critical review. Toxicol Sci 2010; 116: 364-74, https://doi.org/10.1093/toxsci/kfq103.

6. Button TM, Maughan B, McGuffin P. The relationship of maternal smoking to psychological problems in the offspring. Early Hum Dev 2007; 83(11): 727-32, https://doi.org/10.1016/j.earlhumdev.2007.07.006.

7. Cheraghi M, Salvi S. Environmental tobacco smoke (ETS) and respiratory health in children. Eur J Pediatr 2009; 168: 897-905, https://doi.org/10.1007/s00431-009-0967-3.

8. Clemens KJ, Caillé S, Stinus L, Cador M. The addition of five minor tobacco alkaloids increases nicotineinduced hyperactivity, sensitization and intravenous self-administration in rat. Int J Neuropsychopharmacol 2009; 12(10): 1355-66, https://doi.org/10.1017/S1461145709000273.

9. Dempsey DA, Benowitz NL. Risks and benefits of nicotine to aid smoking cessation in pregnancy. Drug Saf 2001; 24: 277-322, https://doi.org/10.2165/00002018-200124040-00005.

10. Donatelle JM. Growth of the corticospinal tract and the development of placing reactions in the postnatal rat. J Comp Neurol 1977; 175: 207-32, https://doi.org/10.1002/cne.901750205. 
11. Durmuş B, Heppe DH, Taal HR, Manniesing R, Raat H, Hofman A, et al. Parental smoking during pregnancy and total and abdominal fat distribution in school-age children: the Generation R Study. Int J Obes (Lond) 2014; 38: 966-72, https://doi.org/10.1038/ijo.2014.9.

12. Ekblad M, Gissler M, Lehtonen L, Korkeila J. Prenatal smoking exposure and the risk for psychiatric morbidity into young adulthood. Arch Gen Psychiatry 2010; 67: 841-49, https://doi.org/10.1001/ archgenpsychiatry.2010.92.

13. Ernst M, Moolchan ET, Robinson ML. Behavioral and neural consequences of prenatal exposure to nicotine. J Am Acad Child Adolesc Psychiatry 2001; 40: 630-41, https://doi.org/10.1097/00004583-200106000-00007.

14. Farkas J, Reglodi D, Gaszner B, Szogyi D, Horvath G, Lubics A, et al. Effects of maternal separation on the neurobehavioral development of newborn Wistar rats. Brain Res Bull 2009; 79: 208-14, https://doi.org/10. 1016/j.brainresbull.2008.12.011.

15. Fox WM. Reflex ontogeny and behavioural development of the mouse. Anim Behav 1965; 13: 234-41, https:// doi.org/10.1016/0003-3472(65)90041-2.

16. Gaszner B, Kormos V, Kozicz T, Hashimoto H, Reglodi D, Helyes Zs. The behavioral phenotype of pituitary adenylate cyclase activating polypeptide deficient mice in anxiety and depression tests is accompanied by blunted c-fos expression in the bed nucleus of the stria terminalis, central projecting Edinger Westphal nucleus, ventral lateral septum and dorsal raphe nucleus. Neuroscience 2012; 202: 283-99, https://doi.org/10. 1016/j.neuroscience.2011.11.046.

17. Gilliland FD, Berhane K, McConnell R, Gauderman WJ, Vora H, Rappaport ED, et al. Maternal smoking during pregnancy, environmental tobacco smoke exposure and childhood lung function. Thorax 2000; 55: 271-6, https://doi.org/10.1136/thorax.55.4.271.

18. Hellström-Lindahl E, Seiger A, Kjaeldkaard A, Nordberg A. Nicotine-induced alterations in the expression of nicotinic receptors in primary cultures from human prenatal brain. Neuroscience 2001; 105(3): 527-34, https://doi.org/10.1016/s0306-4522(01)00209-3.

19. Hill EP, Hill JR, Power GG, Longo LD. Carbon monoxide exchanges between the human fetus and mother: a mathematical model. Am J Physiol 1977; 232: H311-23, https://doi.org/10.1152/ajpheart.1977.232.3.H311.

20. Hill JM, Gozes I, Hill JL, Fridkin M, Brennemann DE. Vasoactive intestinal peptide antagonist retards the development of neonatal behaviors in the rat. Peptides 1991; 12: 187-92, https://doi.org/10.1016/01969781(91)90186-s.

21. Hoffmann D, Hoffmann I. The changing cigarette, 1950-1995. J Toxicol Environ Health 1997; 50: 307-64, https://doi.org/10.1080/009841097160393.

22. Horvath G, Reglodi D, Farkas J, Vadasz G, Mammel B, Kvarik T, et al. Perinatal positive and negative influences on the early neurobehavioral reflex and motor development. In: Antonelli M, editor. Perinatal Programming of Neurodevelopment. Advances in Neurobiology, New York, NY: Springer; 2014, vol 10, p. 149-67.

23. Ikeda T, Mishima K, Yoshikawa T, Iwasaki K, Fujiwara M, Xia YX, et al. Selective and long-term learning impairment following neonatal hypoxic-ischemic brain insult in rats. Behav Brain Res 2001; 118: 17-25, https://doi.org/10.1016/s0166-4328(00)00287-4.

24. Jansen EM, Low WC. Long-term effects of neonatal ischemic-hypoxic brain injury on sensorimotor and locomotor tasks in rats. Behav Brain Res 1996; 78: 189-94, https://doi.org/10.1016/0166-4328(95)00248-0.

25. Jauniaux E, Gulbis B, Acharya G, Thiry P, Rodeck C. Maternal tobacco exposure and cotinine levels in fetal fluids in the first half of pregnancy. Obstet Gynecol 1999; 93: 25-9, https://doi.org/10.1016/s0029-7844(98) 00318-4.

26. Johnson LC, Parker K, Aguirre BF, Nemkov TG, D'Alessandro A, Johnson SA, et al. The plasma metabolome as a predictor of biological aging in humans. Geroscience 2019; 41(6): 895-906, https://doi.org/10.1007/ s11357-019-00123-w. 
27. Kirkpatrick AC, Stoner JA, Donna-Ferreira F, Malatinszky GC, Guthery LD, Scott J, et al. High rates of undiagnosed vascular cognitive impairment among American Indian veterans. Geroscience 2019; 41(3): 351-61, https://doi.org/10.1007/s11357-019-00055-5.

28. Kiss P, Hauser A, Tamas A, Lubics A, Racz B, Horvath Z, et al. Development of neurological reflexes and motor coordination in rats neonatally treated with monosodium glutamate. Neurotox Res 2005; 8: 235-44, https://doi.org/10.1007/bf03033977.

29. Kvarik T, Mammel B, Reglodi D, Antonelli MC, Farkas J, Tamas A, et al. Effects of maternal stress during different periods of pregnancy on the early neurobehavioral response of rats. J Neurol Neurosci 2016; 7: 80.

30. Law KL, Stroud LR, LaGasse LL, Niaura R, Liu J, Lester BM. Smoking during pregnancy and newborn neurobehavior. Pediatrics 2003; 11: 1318-23, https://doi.org/10.1542/peds.111.6.1318.

31. Lee BE, Hong YC, Park H, Ha M, Kim JH, Chang N, et al. Secondhand smoke exposure during pregnancy and infantile neurodevelopment. Environ Res 2011; 111: 539-44, https://doi.org/10.1016/j.envres.2011.02.014.

32. Lubics A, Reglodi D, Tamas A, Kiss P, Szalai M, Szalontay L, et al. Neurological reflexes and early motor behavior in rats subjected to neonatal hypoxic/ischemic injury. Behav Brain Res 2005; 157: 157-65, https:// doi.org/10.1016/j.bbr.2004.06.019.

33. Luck W, Nau H, Hansen R, Steldinger R. Extent of nicotine and cotinine transfer to the human fetus, placenta and amnionic fluid of smoker mothers. Dev Pharmacol Ther 1985; 8: 384-95, https://doi.org/10.1159/ 000457063.

34. Marco EM, Macrí S, Laviola G. Critical age windows for neurodevelopmental psychiatric disorders: evidence from animal models. Neurotox Res 2011; 19(2): 286-307, https://doi.org/10.1007/s12640-010-9205-Z.

35. Navarro HA, Seidler FJ, Eylers JP, Baker FE, Dobbins SS, Lappi SE, et al. Effect of prenatal nicotine exposure on development of central and peripheral cholinergic neurotransmitter systems: evidence for cholinergictrophic influences in developing brain. J Pharmacol Exp Ther 1989; 251(3): 894-900.

36. Peters MA, Ngan LL. The effects of gestational exposure to nicotine on pre- and postnatal development in the rat. Arch Int Pharmacodyn Ther 1982; 257: 155-67.

37. Reglodi D, Atlasz T, Szabo E, Jungling A, Tamas A, Juhasz T, et al. PACAP deficiency as a model of aging. Geroscience 2018; 40(5-6): 437-52, https://doi.org/10.1007/s11357-018-0045-8.

38. Reglodi D, Kiss P, Tamas A, Lengvari I. The effects of PACAP and PACAP antagonists on the neurobehavioral development of newborn rats. Behav Brain Res 2003; 140: 131-9, https://doi.org/10.1016/s01664328(02)00289-9.

39. Rousset CI, Kassem J, Aubert A, Planchenault D, Gressens P, Chalon S, et al. Maternal exposure to lipopolysaccharide leads to transient motor dysfunction in neonatal rats. Dev Neurosci 2013; 35(2-3): 172-81, https://doi.org/10.1159/000346579.

40. Salmon AB, Dorigatti J, Huber HF, Li C, Nathanielsz PW. Maternal nutrient restriction in baboon programs later-life cellular growth and respiration of cultured skin fibroblasts: a potential model for the study of agingprogramming interactions. Geroscience 2018; 40(3): 269-78, https://doi.org/10.1007/s11357-018-0024-0.

41. Schneider T, Bizarro L, Asherson PJN, Stolerman IP. Gestational exposure to nicotine in drinking water: teratogenic effects and methodological issues. Behav Pharmacol 2010; 21: 206-16, https://doi.org/10.1097/fbp. 0b013e32833a5bb5.

42. Shea AK, Steiner M. Cigarette smoking during pregnancy. Nicotine Tob Res 2008; 10(2): 267-78, https://doi. org/10.1080/14622200701825908.

43. Smart JL, Dobbing J. Vulnerability of developing brain. II. Effects of early nutritional deprivation on reflex ontogeny and development on behavior in the rat. Brain Res 1971; 28: 85-95, https://doi.org/10.1016/00068993(71)90526-9. 
44. Smart JL, Dobbing J. Vulnerability of developing brain. VI. Relative effects of foetal and earlypostnatal undernutrition on reflex ontogeny and development of behavior in the rat. Brain Res 1971; 33: 303-14, https:// doi.org/10.1016/0006-8993(71)90105-3.

45. Smith LA, Hill CA, Alexander M, Szalkowsky CE, Chrobak JJ, Rosenkrantz TS, et al. Spatial working memory deficits in male rats following neonatal hypoxic ischemic brain injury can be attenuated by task modifications. Brain Sci 2014; 4(2): 240-72, https://doi.org/10.3390/brainsci4020240.

46. Subedi P, Nembrini S, An Q, Zhu Y, Peng H, Yeh F, et al. Telomere length and cancer mortality in American Indians: the Strong Heart Study. Geroscience 2019; 41(3): 351-61, https://doi.org/10.1007/s11357-019-00080-4.

47. Szegeczki V, Bauer B, Jüngling A, Fülöp BD, Vágó J, Perényi H, et al. Age-related alterations of articular cartilage in pituitary adenylate cyclase-activating polypeptide (PACAP) gene-deficient mice. Geroscience 2019; 41(6): 775-93, https://doi.org/10.1007/s11357-019-00097-9.

48. Wadhwa PD, Buss C, Entringer S, Swanson JM. Developmental origins of health and disease: brief history of the approach and current focus on epigenetic mechanisms. Semin Reprod Med 2009; 27: 358-68, https://doi. org/10.1055/s-0029-1237424.

49. Wilson CA, Vazdarjanova A, Terry AV. Exposure to variable prenatal stress in rats: effects on anxiety-related behaviors, innate and contextual fear, and fear extinction. Behav Brain Res 2013; 238: 279-88, https://doi.org/ 10.1016/j.bbr.2012.10.003.

50. Yochum C, Doherty-Lyon S, Hoffman C, Hossain MM, Zellikoff J, Richardson JR. Prenatal cigarette smoke exposure causes hyperactivity and agressive behavior: role of altered catcholamines and BDNF. Exp Neurol 2014; 254: 145-52, https://doi.org/10.1016/j.expneurol.2014.01.016.

51. Zeskind PS, Gingras JL. Maternal cigarette-smoking during pregnancy disrupts rhythms in fetal heart rate. J Pediatr Psychol 2006; 31: 5-14, https://doi.org/10.1038/s41598-017-17301-5.

Open Access statement. This is an open-access article distributed under the terms of the Creative Commons Attribution 4.0 International License (https://creativecommons.org/licenses/by/4.0/), which permits unrestricted use, distribution, and reproduction in any medium, provided the original author and source are credited, a link to the CC License is provided, and changes - if any - are indicated. (SID_1) 